\title{
Terahertz multistatic reflection imaging
}

\author{
Timothy D. Dorney \\ Department of Electrical and Computer Engineering, MS 366, Rice University, Houston, Texas 77251-1892 \\ William W. Symes \\ Department of Computational and Applied Mathematics, MS 134, Rice University, Houston, Texas 77251-1892
}

Richard G. Baraniuk and Daniel M. Mittleman

Department of Electrical and Computer Engineering, MS 366, Rice University, Houston, Texas 77251-1892

Received September 25, 2001; revised manuscript received December 3, 2001; accepted January 22, 2002

We describe a new imaging method using single-cycle pulses of terahertz ( $\mathrm{THz}$ ) radiation. This technique emulates the data collection and image processing procedures developed for geophysical prospecting and is made possible by the availability of fiber-coupled $\mathrm{THz}$ receiver antennas. We use a migration procedure to solve the inverse problem; this permits us to reconstruct the location, the shape, and the refractive index of targets. We show examples for both metallic and dielectric model targets, and we perform velocity analysis on dielectric targets to estimate the refractive indices of imaged components. These results broaden the capabilities of $\mathrm{THz}$ imaging systems and also demonstrate the viability of the $\mathrm{THz}$ system as a test bed for the exploration of new seismic processing methods. (C) 2002 Optical Society of America

OCIS codes: $320.0320,100.3010,100.3190$.

\section{INTRODUCTION}

The development of terahertz $(\mathrm{THz})$ "T-ray" imaging in the mid-1990s ${ }^{1}$ generated a great deal of interest, as this technology can be used for a wide range of problems involving remote monitoring and inspection. ${ }^{2,3}$ The unique capabilities of the $\mathrm{THz}$ time-domain spectrometer have also inspired numerous explorations of new imaging techniques for $\mathrm{THz}$ radiation, including time-of-flight reflection imaging and ranging, ${ }^{4-6}$ emission imaging, ${ }^{7}$ nearfield techniques for improved spatial resolution, 8,9 interferometric imaging for improved depth resolution, ${ }^{10}$ and near-real-time display-mode imaging. ${ }^{11}$ More recently, the availability of fiber-coupled $\mathrm{THz}$ transmitters and receivers has created new opportunities for data acquisition and image formation. ${ }^{12-14}$ For example, Ruffin and co-workers describe a technique in which a $\mathrm{THz}$ wave interacts with a two-dimensional target and is subsequently measured at many different positions. ${ }^{15}$ The data are used to reconstruct the field distribution at the position of the target through backpropagation of the Huygens-Fresnel diffraction kernel, a familiar result from the theory of holography. ${ }^{16}$ We recently described a similar image formation procedure, also using fibercoupled $\mathrm{THz}$ antennas. ${ }^{17}$ This approach, known as Kirchhoff migration, ${ }^{18,19}$ borrows not from the optics community but instead from the field of seismic prospecting.

Here, we describe in detail this novel method for generating images using $\mathrm{THz}$ pulses. Because of the strong similarities between subpicosecond $\mathrm{THz}$ pulses and the seismic impulses used for geophysical studies, we are able to employ a mature set of algorithms for image formation. These algorithms are based on much of the same underlying physics as that for the more familiar electromag- netic propagation, in the sense that both are direct consequences of the description of wave propagation using Green's functions. In our case, some simplifying assumptions can be made because the propagating wave is in the form of a broadband transient. In essence, the migration approach places more significance on the travel time than on the amplitude of the measured wave. This approximation neglects important aspects of the collected data, so it necessarily reduces the quality of the generated image. It leads, however, to an imaging algorithm that is simple to implement and is extremely robust against losses due to scattering or absorption of the propagating wave. For this reason, it is well suited for image formation in situations where the $\mathrm{THz}$ wave must propagate through a lossy or disordered medium either before or after interacting with the target. A situation of this sort would, of course, be challenging to handle by using the conventional approach. ${ }^{15}$

In addition to broadening the scope of $\mathrm{THz}$ imaging capabilities, this work also offers new possibilities for the development of novel seismic processing algorithms. Generally, such advances in signal processing techniques are evaluated by using simulated data, for which the effects of scattering or noise can be difficult to emulate. Another option in the testing of new imaging algorithms is to use real data from field studies. In such cases, however, the "correct" answer is often not known with high precision, so comparisons between the new imaging algorithms and the conventional methods are not always revealing. A third option is the use of scale models as test beds, an approach that can provide a valuable new perspective. Physical models have found extensive use in the geophysics community, both for the validation of new 
imaging algorithms and for the study of complex structures. ${ }^{20-22}$ The majority of these have been seismic, and there have been few considerations of geophysical test beds based on electromagnetic propagation. The results presented here demonstrate the feasibility of using few-cycle $\mathrm{THz}$ electromagnetic impulses as models for seismic image formation. Of course, there are a number of important differences between acoustic and electromagnetic waves, the most significant involving the issue of longitudinally polarized waves. Nevertheless, there are many commonalties as well, including such phenomena as refraction, diffraction, and Snell's law. ${ }^{19}$ Also, unlike seismic waves, the wave front of the free-space $\mathrm{THz}$ radiation can be easily manipulated by using lenses or other bulk optical components. Finally, the use of electromagnetic radiation eliminates the issue commonly encountered in the use of acoustic transducers, involving the strong sensitivity to the contact conditions, which is due to the strong impedance mismatch between the transducer and the surrounding medium. As a result, one might expect to learn a great deal from tabletop model systems, such as that described here. In this work, we discuss the method of Kirchhoff migration as applied to $\mathrm{THz}$ imaging. In Section 2, we review the basic principles of the migration approach and our experimental arrangement. In Section 3, we discuss issues of resolution, as well as the optimization of the data acquisition. This is most readily understood in the context of reflective (metallic) targets, for which we show several illustrative examples. Section 4 deals with the imaging of dielectric, rather than metallic, targets. As with many inverse problems, there is a commonly encountered ambiguity in images for which the wave velocity is not known a priori. The seismic community has developed a number of sophisticated metrics for determining the optimal velocity maps in these cases. We discuss the use of the most appropriate of these metrics for the $\mathrm{THz}$ imaging problem.

\section{TWO-DIMENSIONAL MIGRATION}

Seismic data collection is generally arranged to acquire data at the surface. An acoustic pulse is propagated into the subsurface strata, and reflected waves are recorded by an array of receivers. The task in migration is to transform this data set, knowing only the position of the transmitter and the receivers and the travel times of the reflected pulse, into a useful image of the subsurface. In other words, given that the horizontal surface is the $x$ axis and the depth is the $z$ axis, we wish to transform data in the $(x, t)$ domain into the $(x, z)$ domain. We review the theoretical and experimental foundations of migration. Schneider provides a useful guide from both the acoustic and optical perspectives, which we closely follow in our discussion. ${ }^{23}$

The theoretical foundation for the migration transform assumes a point source, generating a spherical wave that propagates into a homogeneous medium. This wave is reflected back to a boundary at the recording surface $z=0$. From first principles, the scalar wave equation governs the propagation of the wave. Given that we can observe the wave along the surface, the solution for the surface integral of the wave equation is desired. Several
Green's functions have been suggested that solve this problem. $^{23}$ The analysis is based on Huygen's principle with the solution commonly called the Kirchhoff integral.

Although we have not restated the mathematics of the transform, a more easily understood explanation can be formulated from the geometry of the experimental arrangement. In Fig. 1(a), a single transmitter with an array of symmetrically placed receivers is located at the recording surface. The emitted spherical pulse propagates downward until a portion of it interacts with a point diffractor, generating a reflected wave. Given that the point scatterer is located at $\left(x_{0}, z_{0}\right)$, the transmitter is at $(0,0)$, and a receiver is at $(x, 0)$, the travel time in a homogeneous medium based on the Pythagorean theorem is

$$
D(x)=v_{0} \tau=\left(x_{0}^{2}+z_{0}^{2}\right)^{1 / 2}+\left[\left(x-x_{0}\right)^{2}+z_{0}^{2}\right]^{1 / 2},
$$

where $v_{0}$ is the velocity in the medium, $\tau$ is the two-way travel time, and $D(x)$ is the total distance from transmitter to target to receiver.

In Fig. 1(b), a collection of waveforms from various receiver positions is shown so that the temporal axes run vertically. In light of Eq. (1), we are not surprised to find that the peaks of these waveforms follow a hyperbolic shape. ${ }^{19}$ Kirchhoff migration uses this fact to transform the collection of waveforms back to the location of the point diffractor. In practice, we use a grid of possible reflector locations. For each point, the expected hyperbola is calculated, and the amplitude from each waveform at the proper time offset is summed. The Kirchhoff summation is then placed at the grid point. Correctly guessed points yield a large summation value, since they traverse through the peaks of multiple waveforms. Incorrectly
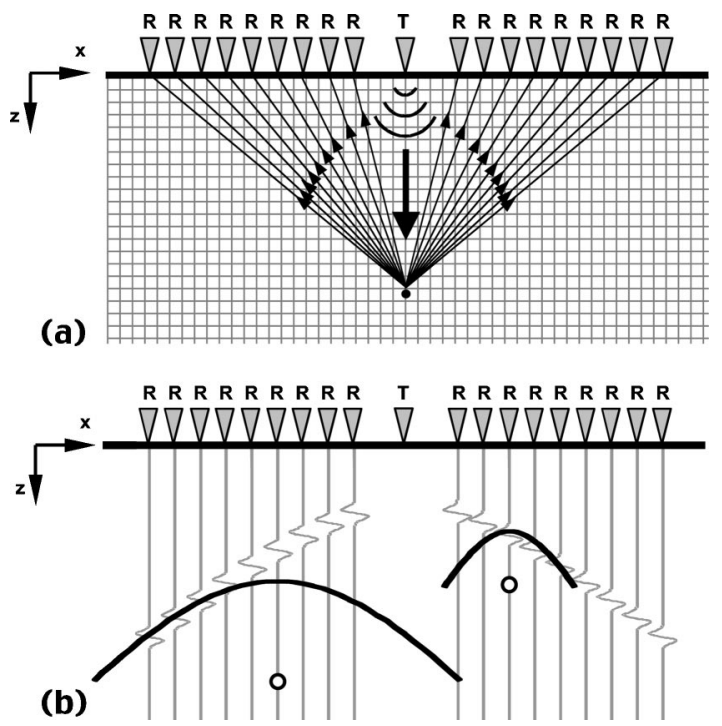

Fig. 1. (a) Schematic of a common shot experimental arrangement emulated by terahertz (THz) system. Multiple symmetrically placed receivers are arranged to collect a series of reflected waveforms from a point scatterer. The travel time increases hyperbolically with the receiver offset from the transmitter. (b) Kirchhoff migration reconstructs the location and the shape of a reflector by calculating the appropriate hyperbola and summing the values of the recorded traces along that hyperbola, for an array of guessed locations. Incorrect locations generate a small summation, since their hyperbolas do not pass through many reflected pulses. Two such incorrect guesses, and their corresponding hyperbolas, are shown. 
guessed points result in smaller values, which is due to the low amplitude of the traversed waveforms and/or the destructive interference from different waveforms in the summation.

Two incorrectly guessed points are shown as circles in Fig. 1(b). The hyperbolas associated with each of these points are centered above the circles. Both points have a small-valued summation, which is due to partial destructive interference. Some of the temporal waveforms have negative amplitude values at the points where they intersect the hyperbola, while others have positive amplitude values at the intersection points. The right-hand point is closer to the surface $(z=0)$; therefore its associated hyperbola has more curvature than the one on the left. These examples illustrate how even incorrect locations can generate nonzero amplitudes in the migration procedure and induce image artifacts. The characteristics of these artifacts are seen in several of the figures below. As the number of waveforms included in the summation increases, the amplitude of these artifacts decreases and the effects become less severe.

From the mathematical outline by Schneider, we note three major differences between theory and practice. ${ }^{23}$ First, the Kirchhoff surface integral is a continuous function, whereas practical implementation requires discrete summation to approximate the integral on account of a finite number of receivers. Discrete summation introduces the need to prevent aliasing effects, which we discuss in Section 3. Second, Kirchhoff migration, strictly speaking, takes into account the reduction in amplitude of the wave as the transmitter-to-receiver distance increases. In our work, the transmitter-to-receiver distance is small enough for us to neglect this effect, given the negligible absorption of the intervening medium. The process of Kirchhoff migration without corrections for the relative change in amplitude between received waveforms is known as diffraction summation as opposed to Kirchhoff migration, but these terms are often used interchangeably within the seismic community. ${ }^{24}$ We follow this trend and generally refer to our approach as Kirchhoff migration. Third, our waveforms are discrete time recordings. We must therefore employ interpolation when the intersection with a hyperbolic curve falls between two time steps.

Kirchhoff migration provides a relatively simple means to reconstruct an image of a point diffractor from reflected waveforms. In general, we can also image extended objects, since any surface may be represented as a collection of point scatterers. The ability to image an object, even if it contains surfaces tilted with respect to the incoming beam, emphasizes one unique feature of this imaging technique. With conventional $\mathrm{THz}$ reflection measurements, even those using a bistatic configuration, ${ }^{6,25}$ a surface with a tilt at a large angle does not reflect any radiation back to the receiver. The radar community has understood the advantages of multistatic over bistatic imaging for many years. ${ }^{26}$

Another unique aspect of Kirchhoff migration is the ability to estimate the parameters of a dielectric target such as thickness and refractive index. To properly create a depth image from temporal signals, we must also know the velocity of the media. We apply one such veloc- ity analysis technique in Section 4. In fact, it should be possible to estimate velocity parameters for a complex two-dimensional dielectric target, since Kirchhoff migration handles lateral velocity changes better than competing methods. ${ }^{19}$ There are fundamental limits, however, to the resolution of the reconstruction in both the horizontal ( $x$-axis) and vertical ( $z$-axis) directions. We examine these limits and discuss the requirements of the experimental arrangement in Section 3.

The $\mathrm{THz}$ time-domain spectrometer used in our measurements is identical to the fiber-coupled system described previously. ${ }^{12,14,15}$ Figure 2 shows a representation of the experimental configuration. The system includes dispersion compensation to offset the effects of the optical fiber and a separate enclosure for fiber splitting, delay rail, and data acquisition. The emitted radiation reflects off a target and is sensed by a receiver. The receiver is a conventional photoconductive antenna, coupled to an $R=4 \mathrm{~mm}$ aplanatic hyperhemispherical substrate lens. Although we are collecting these waveforms serially, by repositioning a single receiver at many different locations, this process simulates simultaneous multistatic data acquisition. Since the receiver is fiber coupled, repositioning does not alter the relative temporal delay of the laser arrival time or the alignment of the femtosecond optical beam onto the receiver. We translate the receiver along a line and rotate the antenna to compensate for its angular sensitivity. ${ }^{14}$ Great care is taken to ensure that the axis of this rotation passes through the antenna, so that the rotation does not introduce any temporal shifts in the measured waveforms. This configuration mimics the typical situation in geophysical imaging, where the transmitter is a device for generating a seismic impulse (e.g., dynamite) and the receivers are an array of geophones. ${ }^{19}$

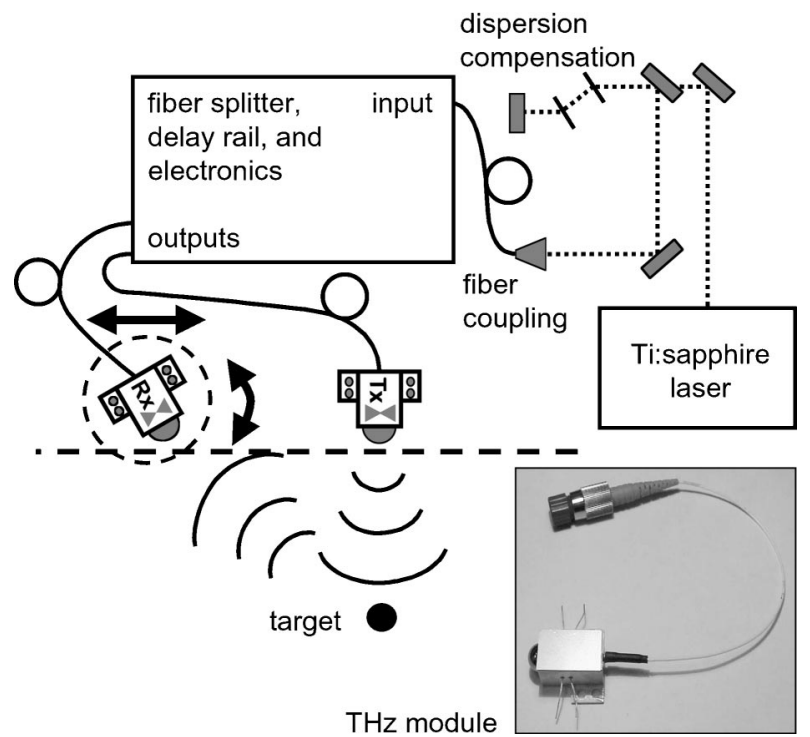

Fig. 2. We acquire data by using the Picometrix fiber-coupled $\mathrm{THz}$ system that provides a constant temporal delay as the transmitter and/or the receiver is translated. The system includes dispersion compensation to offset the effects of the optical fiber and a separate enclosure for fiber splitting, delay rail, and data acquisition. To simulate simultaneous multistatic data acquisition, we translate and rotate the receiver along a line. A photograph of a packaged antenna module is shown. 


\section{RESOLUTION OF RECONSTRUCTED IMAGES}

As discussed in Section 2, Kirchhoff migration provides a means for reconstruction of a point scatterer from temporal data in a homogeneous medium. This method also can reconstruct an arbitrary shape, since any surface may be regarded as a collection of point scatterers. Limitations exist as to how well the reconstructed image is resolved. We discuss the theoretical limits and demonstrate the effects with both experimental and simulated data.

Horizontal resolution defines the smallest feature that can be resolved along the $x$ dimension (parallel to the array of receivers). It is generally defined in terms of the first Fresnel zone. Generally, the first Fresnel zone is defined by the size of an opening in an infinite plate such that only positive values of an incident spherical wave are able to penetrate the opening. ${ }^{16,18}$ For a broadband source, the Fresnel zone can be defined by the size of an aperture that maximizes the transmitted energy. ${ }^{27}$ The size of the first Fresnel zone is given approximately by

$$
\Delta x=\frac{v_{0}}{2} \sqrt{\frac{\tau}{f_{\text {mean }}}},
$$

where $f_{\text {mean }}$ is the mean frequency of the $\mathrm{THz}$ source and $v_{0}$ and $\tau$ are as defined in Eq. (1). ${ }^{19}$

A feature that is smaller than the size of the first Fresnel zone cannot be resolved. As the feature size increases beyond the size of the first Fresnel zone, the image reconstruction provides a better representation. This viewpoint is equivalent to asking how much the resolution improves as the hole in our infinite plate expands. As the size of the hole increases, more of the spherical wave is allowed to traverse the opening. The field from each subsequent Fresnel zone contributes to the net field with sign opposite to that of the field from the preceding zone. As additional Fresnel zones are available, the resolution therefore improves, but, at the same time, the effect diminishes as more zones are added. ${ }^{28}$

Vertical resolution defines the smallest feature that can be resolved along the $z$ dimension (the initial beam propagation direction). This depends on the coherence length of the probing wave. A shift in position of a temporal waveform by an amount that is small compared with the duration of the $\mathrm{THz}$ pulse will not have a significant effect on the summation. Consequently, the migration summation is not sensitive to temporal shifts of this magnitude. The generated images exhibit blurred edges, which are due to the finite coherence length of the radiation. Vertical resolution is given by

$$
\Delta z=\frac{v_{0}}{4 \Delta f},
$$

where $\Delta f$ is the bandwidth of the radiation.

We demonstrate the limits of both horizontal and vertical resolution with an experiment using long metal cylinders of various diameters. These cylinders may be taken to be ideal reflectors. Each cylinder is placed approximately $90 \mathrm{~mm}$ from a fixed $\mathrm{THz}$ transmitter. As in Fig. 1(a), a series of 152 reflected waveforms are collected on either side of the transmitter in 1-mm steps, which is somewhat smaller than the effective aperture of the receiver antenna. The smallest transmitter-to-receiver offset is $38 \mathrm{~mm}$, limited by the size and the orientation of the antenna housings. Images are formed from the resulting set of waveforms by using the migration procedure outlined above. These results are shown in Fig. 3 for five cylindrical targets with various diameters. The vertical axes show the distance from the transmitter, while the dashed circles show the actual positions and cross sections of the targets. For all five images, we used a grid spacing (pixel size) of $50 \mu \mathrm{m}$ for the image reconstruction. The migration results place the targets at the proper position within our ability to independently determine them.

We note that the two smallest cylinders are accurately placed; however, they are not well resolved. The diameters of these cylinders are very close to the horizontal resolution. For our measurements, the size of the first Fresnel zone is $\Delta x \sim 2.9 \mathrm{~mm}$. For the $4.7-\mathrm{mm}$-diameter cylinder, we begin to resolve features in the image that correspond with the location of the reflecting surface. The two largest cylinders have a clearly defined surface section. Only a portion of the surface is imaged, on account of the finite range of receivers along the $x$ axis.

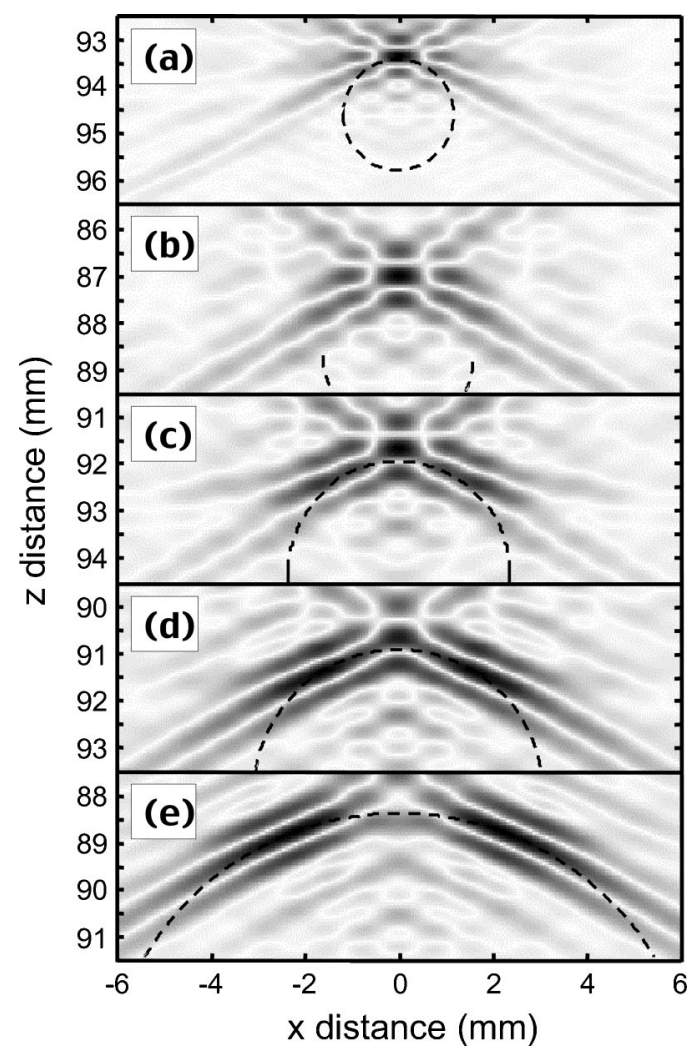

Fig. 3. Kirchhoff migration images from five metal cylinder targets with diameters of (a) $2.4 \mathrm{~mm}$, (b) $3.2 \mathrm{~mm}$, (c) $4.7 \mathrm{~mm}$, (d) 6.2 $\mathrm{mm}$, and (e) $12.7 \mathrm{~mm}$. The dashed curves represent the outlines of the targets for comparison with the migration images. In (a) and (b), the objects are correctly located, but their surface curvature cannot be resolved, since their diameters are close to the resolution. In (c), the location of the object and a very faint surface curvature are resolved. In (d) and (e), the cylindrical surfaces are reconstructed over a limited region on account of the finite range of receiver offsets. 
The limited number of receivers hinders complete cancellation of diffraction sums from regions without a reflector. Consequently, a number of image artifacts arising from aliasing effects are observed, as anticipated above. These artifacts blend into the surface reconstruction at its outer limits and appear to simply continue the surface along a tangent line on either side of the cylinder. The angles of these artifacts are determined both by the distance from the transmitter to the object (which determines the curvature of the hyperbolic reflection curve) and by the shape of the reflecting object. The reconstructed curves of the cylindrical surfaces also exhibit a finite thickness. The finite coherence length of the radiation, which limits the vertical resolution, causes blurring that is 3-4 pixels wide. ${ }^{18}$ The surface blurring is consistent with the calculated vertical resolution of $\Delta z$ $\sim 0.19 \mathrm{~mm}$.

The experimental arrangement may also have a large effect on the reconstructed image. Obviously, the resolution limits are determined by the bandwidth of the $\mathrm{THz}$ pulse, but increased number of artifacts and lack of detail in complex shapes may result if the experimental arrangement is not properly designed. Two important concerns are the receiver's range, or span across the surface, and the receiver's spacing.

Receiver span determines the angle of a target that can be observed. Simple geometry provides sufficient insight, and the reconstructed cylinder images in Fig. 3 provide a useful illustration. With a receiver offset from 38 to $189 \mathrm{~mm}$ on either side of the transmitter, the observed section of the cylinder's curvature is limited. Increasing the receiver's span allows more of the target to be reconstructed but at the expense of additional acquisition and processing time.

Given the desire to acquire data over a broader range of receiver positions, it might seem obvious to simply increase the step size between receiver positions. Diffraction sums, however, rely not only on coherent summation to define where an object exists but also on destructive summation of incorrectly guessed hyperbolas. As the receiver spacing increases, the ability to create destructive interference by using Kirchhoff migration diminishes. In fact, a receiver spacing that creates a shift in the temporal waveforms greater than half of the temporal duration of the pulse effectively destroys the ability to properly resolve the image. Equally, the receivers must be arranged so that such a shift does not occur between any pair of adjacent receivers. Complex targets may have a large effect on the temporal shift of the acquired waveforms. We define the upper bound of the stepping distance in terms of the signal bandwidth $\Delta f$ :

$$
\Delta D_{n}=\left|D\left(x_{n}\right)-D\left(x_{n+1}\right)\right| \leqslant \frac{v_{0}}{2 \Delta f}
$$

where $D\left(x_{n}\right)$ is the transmitter-to-target-to-receiver distance of adjacent receivers and $v_{0}$ is the velocity of the wave in the homogeneous medium. For simple structures, this provides a means to determine the receiver spacing. For example, assume that a flat reflector, parallel to the transmitter/receiver line, is located $90 \mathrm{~mm}$ from the surface. A receiver position step size of $1 \mathrm{~mm}$

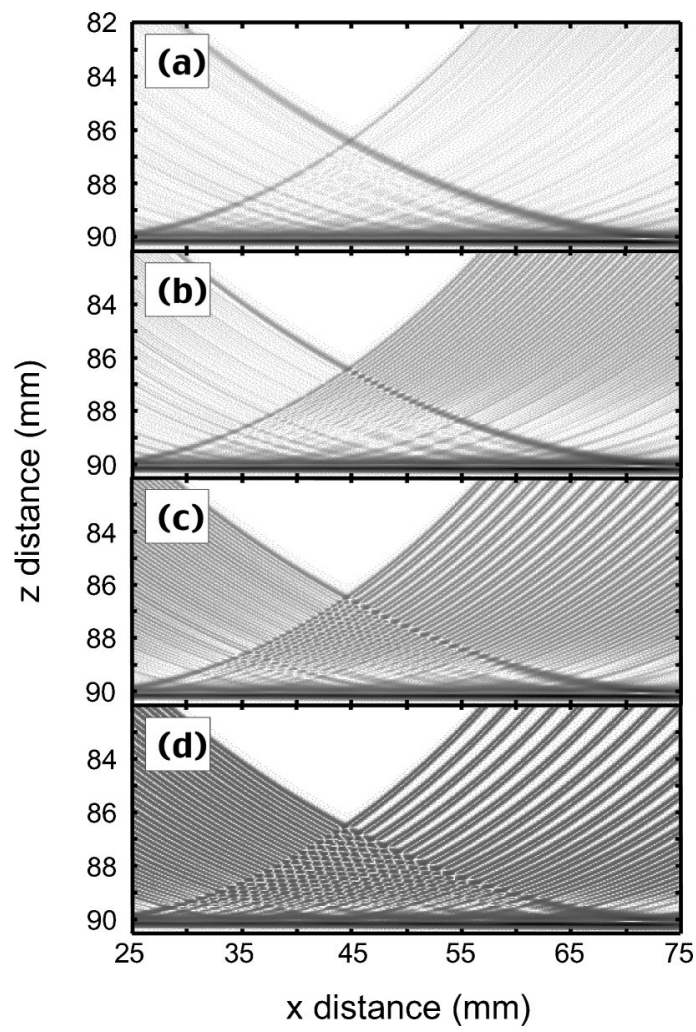

Fig. 4. Reconstruction of a planar reflector using a single transmitter and a group of receivers on one side of the transmitter with different receiver spacings, demonstrating the effect of aliasing. In (a), (b), (c), and (d), the receivers are spaced every 1 , 2,3 , and $4 \mathrm{~mm}$, respectively, within an offset range of 38-157 $\mathrm{mm}$.

(e.g., from 38 to $39 \mathrm{~mm}$ ) results in a difference $\Delta D_{n}$ of $\sim 0.21 \mathrm{~mm}$, which is less than the upper bound of $\sim 0.3$ $\mathrm{mm}$ for a $0.5-\mathrm{THz}$ bandwidth. A stepping distance of 1 $\mathrm{mm}$ is appropriate, and it is used for all experimental data in this paper.

To demonstrate the effects of aliasing due to improper receiver placement, we use simulated data. A flat, reflective plate is located at $z=90 \mathrm{~mm}$ with a single transmitter and 120 receivers positioned on one side of the transmitter at 1-mm spacing. The minimum transmitter-toreceiver offset is $38 \mathrm{~mm}$. Four images were reconstructed by using the data, each with a progressively reduced data set. In Fig. 4(a), we used all 120 waveforms in the migration. Artifacts are apparent but are relatively small compared with the reconstruction for the reflecting plate. For Fig. 4(b), alternate waveforms were discarded (i.e., receivers $1,3,5, \ldots, 119$ were used) before the Kirchhoff migration, while Fig. 4(c) used data from receivers 1, 4, 7,..., 118 and Fig. 4(d) used data from receivers $1,5,9, \ldots, 117$. We observe a dramatic effect as the aliasing artifacts get progressively worse with each increase in receiver stepping distance.

\section{VELOCITY ESTIMATION WITH SEMBLANCE}

In Section 3, we demonstrated the ability of the THz system to image curved surfaces. Imaging steep angles relative to the transmitter is possible with a multistatic 
receiver arrangement. This experiment is a useful illustration but contains only a single homogeneous medium (air). A more interesting problem involves imaging dielectric targets. Dielectrics allow both reflection and transmission of the electromagnetic field and give rise to regions of differing velocities within the reconstructed image. To properly create an image, we require a method to estimate the different velocities. In an effort to emulate seismic data acquisition, we study layered media, a common geologic structure. To illustrate the important steps in image formation, we start with simulated $\mathrm{THz}$ data. These simulations demonstrate the challenges inherent in this image reconstruction problem, which are formidable even without noise or other experimental considerations. Once we have covered the salient points, we then turn to experimental results, using a composite target with a very small impedance mismatch. These data illustrate the sensitivity of the $\mathrm{THz}$ system and the applicability of the velocity estimation procedures outlined below.

For the simulations, a simple planar target is a useful starting point. A single homogeneous layer is oriented parallel to the transmitter/receiver line. Its top surface is at $z=90 \mathrm{~mm}$, and its bottom surface is at $z$ $=100 \mathrm{~mm}$. The regions both above and below this planar target are empty. The refractive index of the simulated material is set to 1.525 , which is roughly equivalent to that of high-density polyethylene (HDPE). ${ }^{29}$ The material is assumed to be nondispersive. Figure 5 shows the data acquisition arrangement, in which a group of one transmitter and 20 receivers is moved across the layer. The initial transmitter-to-receiver offset is $38 \mathrm{~mm}$, with the receiver spacing set at $2 \mathrm{~mm}$. Data from these 20 receivers are collected every $2 \mathrm{~mm}$ at ten locations. We note that seismic data collected at sea are gathered by using a similar technique, where the acoustic source is an air gun followed by a towed streamer of acoustic receivers. ${ }^{19}$

We wish to use the same Kirchhoff (diffraction) summation as that described above to create an image from the entire data set. Previously, we used only common shot

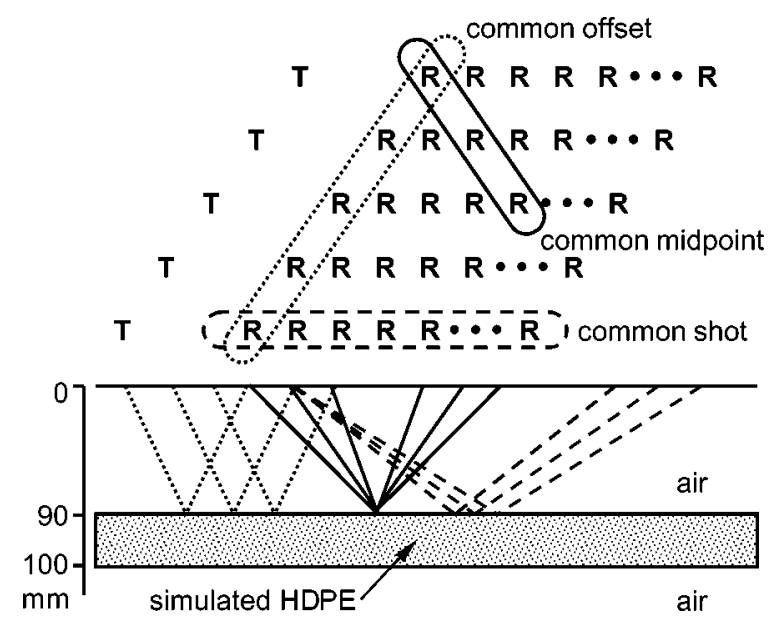

Fig. 5. Data acquisition of a synthetic planar, homogeneous dielectric material using a moving group of one transmitter and 20 receivers. This arrangement allows common offset, common midpoint, and common shot gathers. We use a refractive index of 1.525, similar to that of high-density polyethylene (HDPE).

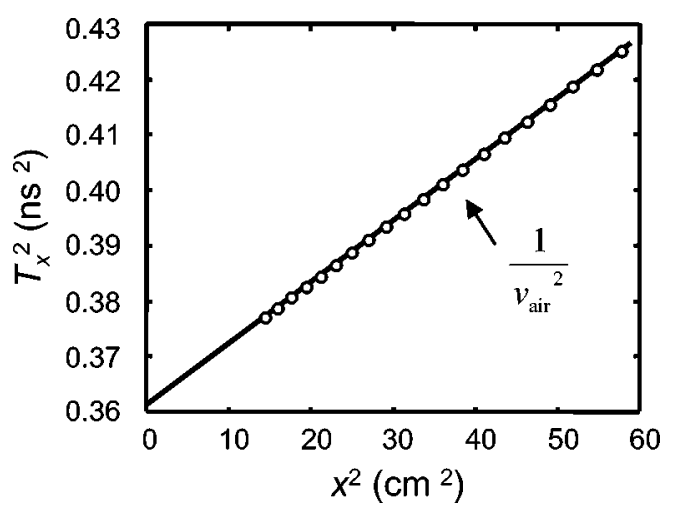

Fig. 6. The absolute propagation time for the THz system is determined by plotting the squared receiver offset for a flat plate while adjusting the squared two-way travel time until the slope equals $1 / v_{\text {air. }}^{2}$ Green's technique ${ }^{28}$ is used to determine the wave velocity $v_{0}$ when the absolute time is known. We know precisely the wave velocity but do not have an absolute time reference.

data, or, in other words, data acquired by a set of receivers from a single transmitter location. We now have several advantageous data arrangements, such as common midpoint and common offset, that can be extracted from multistatic data sets of this type. Common midpoint data allow multiple views of the same spatial location. In effect, we use these various views to reduce noise (e.g., through averaging) or infer structure by noting variations in waveforms not accounted for by the change in transmitter-to-receiver distance. Common offset is useful in determining angle and/or velocity changes as the transmitter-to-receiver offset is fixed. Time-of-flight variations indicate a change in depth or tilt of the reflector, or velocity of the intervening media.

One important difference between the application of Kirchhoff migration to electromagnetic and acoustic scattering is that, in the seismic experiment, the absolute propagation time is known while the wave velocity $v_{0}$ is generally not known. With our data, the wave velocity of the first layer (air) is known precisely, but the time between the emission and the detection of the $\mathrm{THz}$ pulse is not known, since the measurements provide only relative delays. In both cases, however, the unknown quantity can be obtained from Green's equation ${ }^{30}$ :

$$
T_{x}^{2}=T_{0}^{2}+\frac{x^{2}}{v_{0}^{2}},
$$

where $T_{x}$ is the absolute transmitter-to-receiver travel time for a receiver at $(x, 0)$ and $T_{0}$ is the travel time for a (hypothetical) receiver at $x=0$ (the location of the transmitter). ${ }^{30}$ If a flat reflector oriented parallel to the receiver line is used, a plot of $T_{x}^{2}$ versus $x^{2}$ produces a line. If the absolute travel time is known, then the slope of the line determines the wave velocity. In our case, we vary the temporal offset of all waveforms until the slope equals $1 / v_{\text {air }}^{2}$; then the intercept gives the absolute value of $T_{0}$ and therefore the transmitter-to-target distance $\left(z_{0}=v_{\text {air }} T_{0} / 2\right)$. Figure 6 shows the arrival time of the primary reflection from common shot data after the appropriate time shift of the waveforms. We used the absolute time $T_{0}$ to calibrate the time axis in the above cylinder experiments. 
After adjusting the temporal waveforms to an absolute time scale, we perform Kirchhoff migration by using a 50- $\mu \mathrm{m}$ pixel size for the flat plate drawn in Fig. 5 . The results are shown in Fig. 7; as expected, both the front and back surfaces of the plate are visible. We note, however, that the distance between the surfaces of the plate is not equal to the thickness of the sample. This is a result of the assumption, implicit in the migration procedure, that the wave velocity is constant everywhere within the imaged region. The velocity of light within the material is $\sim 33 \%$ slower than in air. Consequently, the surfaces appear to be farther apart than they actually are. This type of image artifact was recognized even in the earliest reports of $\mathrm{THz}$ reflection imaging. ${ }^{6}$ We now examine several means to estimate velocity, with the goal of correcting this artifact.

Several simple but effective methods to determine velocities in geophysical imaging are constant-velocity gathers (CVGs) and constant-velocity stacks (CVSs). ${ }^{19}$ With CVGs, a uniform velocity is assumed for all data (e.g., common midpoint), and the temporal waveforms are drawn side by side, or gathered. Each waveform's time base is adjusted to properly align the data. The adjustment, or moveout, is determined by

$$
\Delta T=T_{x}-T_{0}=\left(T_{0}^{2}+\frac{x^{2}}{v_{g}^{2}}\right)^{1 / 2}-T_{0},
$$

where $T_{0}$ is the travel time for a (hypothetical) receiver at $x=0$ (the location of the transmitter) as in Eq. (5), $v_{g}$ is the guessed constant velocity, and $T_{x}$ is the absolute transmitter-to-receiver travel time for a receiver at $(x, 0)$. The moveout value $\Delta T$ is used to shift the time axis of each reflected waveform. A series of different gathers, each with a slightly different guessed velocity, are plotted side by side on a graph of time versus estimated velocity. Visual trends indicate good velocity estimates. An undercorrected gather (where the guessed velocity $v_{g}$ is too fast) creates downward-tilting curves from flat reflectors (parallel to the line of receivers). Overcorrected gathers produce upward-tilting curves from flat reflectors. By se-

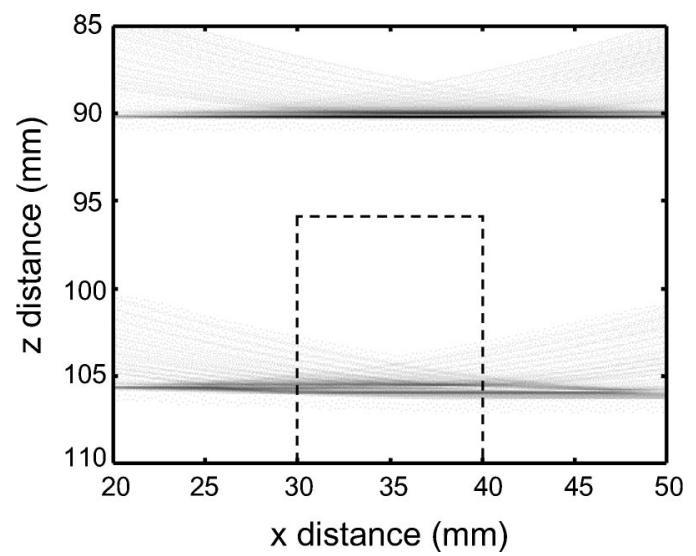

Fig. 7. Kirchhoff (diffraction) summation of the synthetic data collected in Fig. 5 by using a $50-\mu \mathrm{m}$ grid clearly shows the two surfaces of the flat plate. The top surface is accurately placed; however, the $z$-distance separation between the two surfaces is larger than the simulated thickness of $10 \mathrm{~mm}$. Velocity analysis is required and is performed over the region indicated. Figure 8 below displays the results. lecting regions at differing times that have "flat" features, we can determine the correct velocities at each time.

CVSs use a similar approach to that for CVGs, but data are first migrated (summed) across a hyperbola as in Eq. (1). By plotting the stacked (migrated) data, each with a slightly different constant velocity, we look for "flat" regions, which indicate coherent summation (i.e., correct velocities). Typically, CVGs and CVSs are used concurrently with the CVG data taken from one of the data sets used in the CVS. Both of these procedures rely to a great extent on a visual inspection of the data; however, these methods are appropriate for seismic reconstruction, since acoustic velocity generally increases with depth. In fact, a relatively new technique called differential semblance optimization goes so far as to assume a smooth velocity function and moves the discontinuities created by impedance mismatches into a reflectivity function. ${ }^{31}$

Both the CVG and CVS methods assume a root-meansquare (rms) velocity. In other words, if a layered target has velocities $v_{1}, v_{2}, v_{3}, \ldots, v_{n}$ and a one-way travel time of $t_{1}, t_{2}, t_{3}, \ldots, t_{n}$ for each layer, respectively, the rms velocity is defined as ${ }^{19}$

$$
\begin{aligned}
v_{\mathrm{rms}}^{2} & =\frac{v_{1}^{2} t_{1}+v_{2}^{2} t_{2}+v_{3}^{2} t_{3}+\cdots+v_{n}^{2} t_{n}}{t_{1}+t_{2}+t_{3}+\cdots+t_{n}} \\
& =\frac{\sum_{k=1}^{n} v_{k}^{2} t_{k}}{\sum_{k=1}^{n} t_{k}} .
\end{aligned}
$$

Given the sharp velocity discontinuities created as light moves from air to HDPE in our sample target, an average velocity does not provide adequate information. These types of sharp discontinuities are also exhibited in the challenging Marmousi benchmark seismic velocity model..$^{2}$

This still leaves us in need of a method to analyze our simple target. One approach is to break up the velocity function into sections and continue the analysis downward by using an iterative approach, in which the velocity in each layer is determined by using the value from the previous layer. ${ }^{18}$ We apply this approach by using Kirchhoff migration in tandem with a velocity field. The velocity field determines the one-way travel time from a depth point $(z>0)$ to a point on the surface $(z=0)$. The model includes velocities defined over the entire $(x, z)$ space of interest and any deviations due to Snell's law at interfaces. The cumulative depth-point-to-transmitter and depth-point-to-receiver travel times determine the path of the hyperbola used for summation of the data-set values. The velocity model is downward modified as each new interface is encountered.

Figure 8(a) shows the Kirchhoff migration results for the second layer of the simulated target (the layer in which the index is 1.525) at guessed velocities from $2.8 \times 10^{8}$ to $1.8 \times 10^{8} \mathrm{~m} / \mathrm{s}$, corresponding to guessed refractive indices from 1.07 to 1.67, respectively. The firstlayer location and velocity are determined by the results in Fig. 7, since the velocity in the uppermost layer is known. The migration images show a dramatic change 

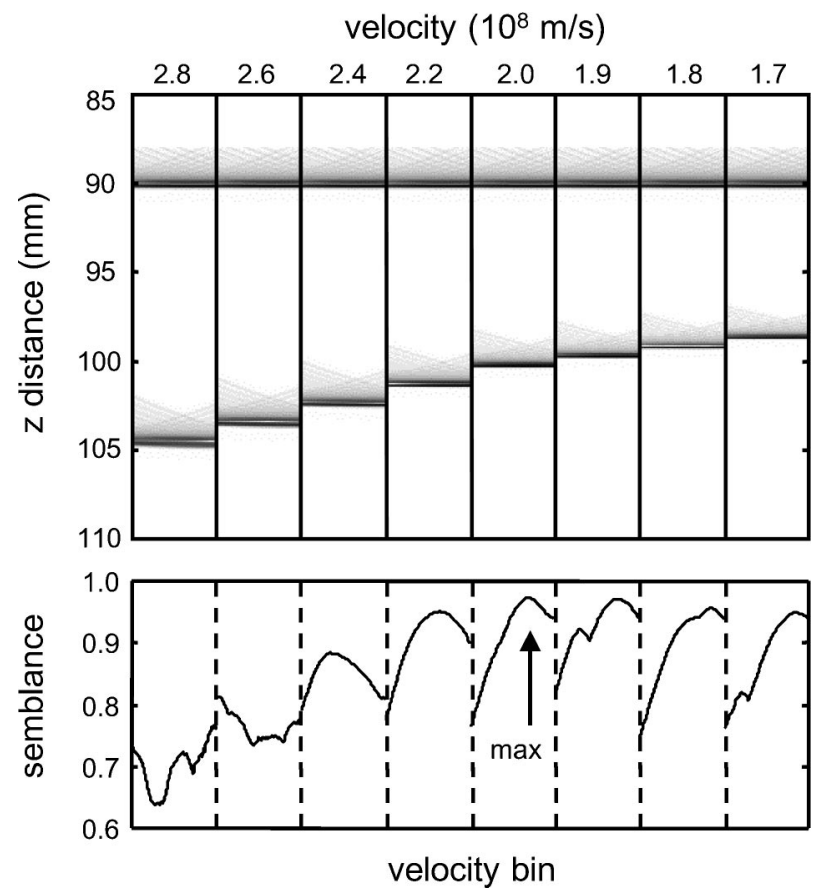

Fig. 8. (a) A comparison of eight different velocity fields applied to the Kirchhoff migration of the second interface in Fig. 7 shows a dramatic change in the placement of the layer. We note a generally decreasing spread of the reconstructed interface as the guessed velocity decreases; however, there is no simple way to distinguish among these guesses. The background has been amplified to display the artifacts. (b) We apply the semblance coherency metric to each column of the migration indicated in Fig. 7 from 95 to $110 \mathrm{~mm}$. The maximum semblance values for each column increase with decreasing velocity but show a curve that is due to the artifacts on both the left and right sides of the reconstruction. The maximum semblance value occurs at $2.0 \times 10^{8} \mathrm{~m} / \mathrm{s}$, close to the actual value of $1.97 \times 10^{8} \mathrm{~m} / \mathrm{s}$.

in the location of the second interface as the guessed velocity in the intervening medium decreases. The background artifacts have been amplified in Fig. 8(a), compared with Fig. 7, to emphasize the differences created by the reconstruction; however, there is no good way to distinguish by inspection between these various results. This ambiguity, arising from the fact that neither the thickness nor the wave velocity is known, is commonly encountered in many different manifestations of the inverse problem. A quantitative measure of the quality of the interface in the migration image is required to distinguish among the various guesses displayed in Fig. 8.

One aspect of these images that can be analyzed to provide this metric is the extent to which the wave fronts align during the migration procedure. Several coherency metrics exist such as velocity spectra, ${ }^{33}$ cross correlation, and semblance. ${ }^{34}$ A velocity spectrum relies on $v_{\text {rms }}$, which, as noted above, will not suffice for this example. Semblance has been shown to differentiate smaller velocity changes than cross correlation ${ }^{34}$; therefore we use semblance as the coherency metric.

The semblance principle can be understood by noting the effect that a change in velocity has on the location of a reflector, as in Fig. 8(a), for example. We create multiple reconstructed images by using different subsets of the data and the same guessed velocity. Since these multiple images are all of the same region of the target, they should all appear identical. This will be true only if the guessed velocity is the correct one. In essence, we create different views of the same target and note the variations from view to view, arising from incorrect velocity estimates. We define an image $I$ generated from Kirchhoff summation as

$$
I(x, z)=\sum_{T}\left[\sum_{R} f(R, t(R, T, x, z))\right],
$$

where the hyperbolic intersection occurs across many waveforms $f$ for a given receiver location $R$ at the time in$\operatorname{dex} t$. The intersection is determined by the location of the transmitter $T$, the location of the receiver $R$, and the subsurface point of interest $(x, z)$. Given that many images are formed from different subsets of the data using the same guessed velocity, we have multiple images $I_{M}(x, z)$. The semblance metric is defined as

$$
S=\frac{\left[\sum_{M} I_{M}(x, z)\right]^{2}}{M \sum_{M} I_{M}^{2}(x, z)},
$$

where all images are pointwise summed, then squared, in the numerator and pointwise squared, then summed, in the denominator. The division by $M$ simply normalizes the metric, so $0 \leqslant S \leqslant 1$.

For the simulated data acquired from Fig. 5, we generated Kirchhoff migration images by using common offset data on a 50- $\mu \mathrm{m}$-square pixel grid. Common offset data reduce the amount of artifacts at the left and right edges of the reconstruction, since it spans a wider range compared with that from common shot data. The reconstructed region from $z=95$ to $110 \mathrm{~mm}$ encompasses the second interface in all velocity estimates in Fig. 8(a). From the 20 images created by using data from ten transmitter/receiver locations for each velocity, we generated a semblance image. The plots in Fig. 8(b) show the maximum semblance value in each column of the semblance images.

The semblance results shown in Fig. 8(b) provide a quantitative metric for selecting the appropriate guessed velocity for the region between the two interfaces. Ideally, the semblance would provide a similar response across the flat secondary interface; however, artifacts, evident in Fig. 8(a), create a reduced semblance on both the left and right sides of the reconstruction, and likewise in the semblance results. These results, however, do indicate that an incorrectly guessed velocity positions the reconstructed interface at different locations depending on the receiver offset from the transmitter. Semblance records the lack of coherency as a lower normalized output-to-input energy ratio. The guessed velocity of $2.0 \times 10^{8} \mathrm{~m} / \mathrm{s}$ has the maximum semblance, while the actual velocity in this simulated target is $1.97 \times 10^{8} \mathrm{~m} / \mathrm{s}$. Next, we want to extend these results to more complex targets.

The previous simulated example was simple and could have been handled by several less computationally expensive methods. Specifically, Dix provides a means to solve for an arbitrary number of velocity intervals. ${ }^{35}$ If this 
analysis method is used, any errors on earlier or later interfaces do not affect subsequent velocity calculation. This seems to be an ideal approach; however, application of this method to highly complex targets quickly becomes untenable, as the analyst must apply simple geometric shapes to represent complex reflectors.

Although continuous downward modifications will be needed to update the velocity field with respect to angled reflectors, multiple layers, synclines (concave-up features), and anticlines (concave-down features or humps), we continue to use Kirchhoff migration and semblance. Kirchhoff migration (diffraction sums) performs well even with shifts in lateral velocity $v(x)$ (Ref. 19), and semblance is a more robust coherency measure. ${ }^{34}$

To illustrate these points, we study a composite dielectric target with substantially smaller refractive-index discontinuities than those in the simulated example described above, using the $\mathrm{THz}$ spectrometer. The experimental target is designed to mimic layered strata common in the geophysics community. The only a priori information used in the analysis is the refractive index of the initial layer, which is air, and the transmitter and receiver positions. This approach follows the work of Neidell and Taner. ${ }^{34}$
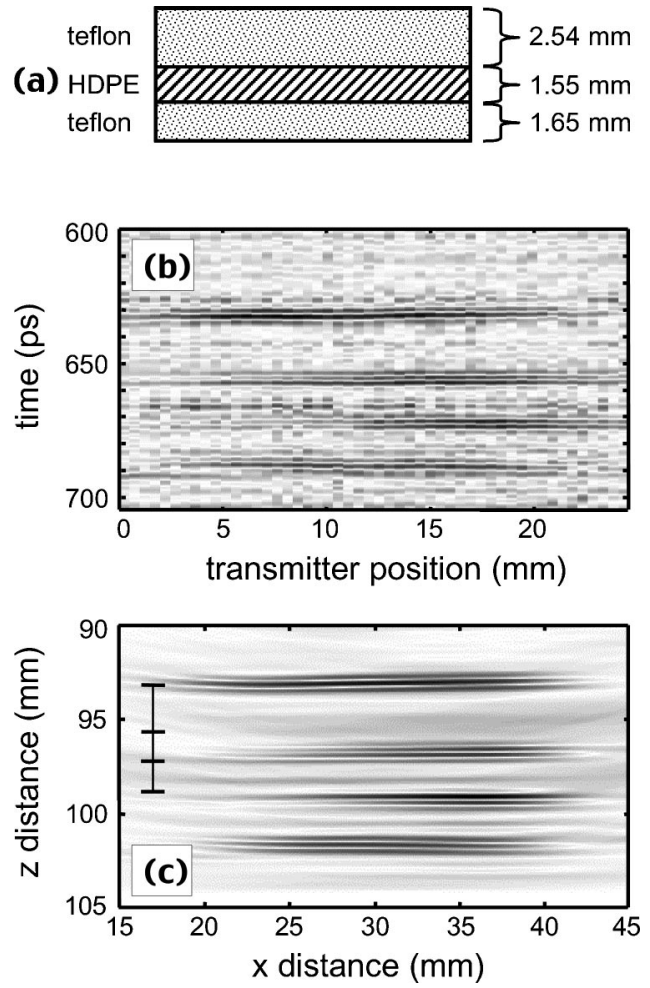

Fig. 9. (a) Construction of an experimental, composite target includes a top layer of Teflon followed by high-density polyethylene (HDPE) and another layer of Teflon. The second and third layers are machined to have the same optical delay at normal incidence. This stack creates a smaller change in velocity between layers compared with that of the simulated air/HDPE interface in Fig. 5. (b) Common offset data after filtering show a large amount of noise. The interfaces are hard to discern; however, the constant-velocity migration results in (c) dramatically reduce the noise and reconstruct the image. The indicators at left denote the expected locations of each interface. These demonstrate the need for velocity analysis to properly locate the interfaces.
Figure 9(a) shows a schematic of the composite target. Only general dimensions are displayed, since several irregularities from machining exist. The target is composed of a top layer of Teflon. Two additional plates, one of HDPE and the second of Teflon, are compressed onto the top plate. The difference in velocities between Teflon and HDPE is only $\sim 6 \%$, compared with the $30 \%$ difference between Teflon and air. ${ }^{29}$ Furthermore, the thickness of the bottom Teflon plate is machined to $1.65 \mathrm{~mm}$, while that of the HDPE plate is $1.55 \mathrm{~mm}$. With these thicknesses, the bottom two plates induce the same optical delays at normal incidence. The goals of this experiment are to correctly locate the four interfaces and also to identify the composition of each layer by accurately determining its refractive index using the semblance analysis.

To analyze this composite target, we translated one transmitter and 19 receivers over the target. The initial receiver offset was approximately $34 \mathrm{~mm}$, and the receiver spacing was $1 \mathrm{~mm}$. The group of transmitters and receivers acquired data at 25 positions with a $1-\mathrm{mm}$ spacing for a total of 475 acquired $\mathrm{THz}$ waveforms. Figure 9(b) shows one of the common offset gathers with the corrected temporal offset. The first flat region of the target was used to calculate the absolute temporal offset with the method described above for Eq. (5). The interfaces are a bit hard to discern because of the small amplitude of the reflections from the nearly impedance-matched interfaces.

Figure 9(c) shows the Kirchhoff migration, assuming a constant velocity throughout the imaged region. As above, the pixel size is $50 \mu \mathrm{m}$. From this migration, the four interfaces are clearly displayed. The noise is dramatically reduced compared with that in Fig. 9(b). The four marks superimposed on the figure show the actual locations of the interfaces. The first interface is correctly located because the index of the intervening medium (air) is known; however, the remaining interfaces are not correctly located because of the nonuniform velocity field. As in Fig. 8, we require velocity analysis to properly locate these buried interfaces.

We apply the semblance analysis not to the entire image but instead to a small region within the image. To determine the velocity below the first interface, we use a small analysis window that encompasses the interface of interest. In this example, the window size is chosen to be $3 \mathrm{~mm}$ high and approximately $1.5 \mathrm{~mm}$ wide. Each interface is analyzed in turn, starting with the layer closest to the top (known) interface. We guess a constant velocity below the previous interface and compute the semblance at each pixel within this window. The semblance, averaged over all pixels in the window, is shown in Fig. 10(a) as a function of the guessed velocity. The maximum average semblance determines the correct velocity in the first dielectric layer. This process is then repeated at the third interface (bottom of the second dielectric layer) by using the (now known) velocity of the first layer to determine the velocity of the second layer. The process is iterated once more to determine the velocity in the third layer. The average semblance values for each layer are shown in Fig. 10.

Unlike the simulated results in Fig. 8(b), noise and multiple reflections at the interfaces create a challenge 

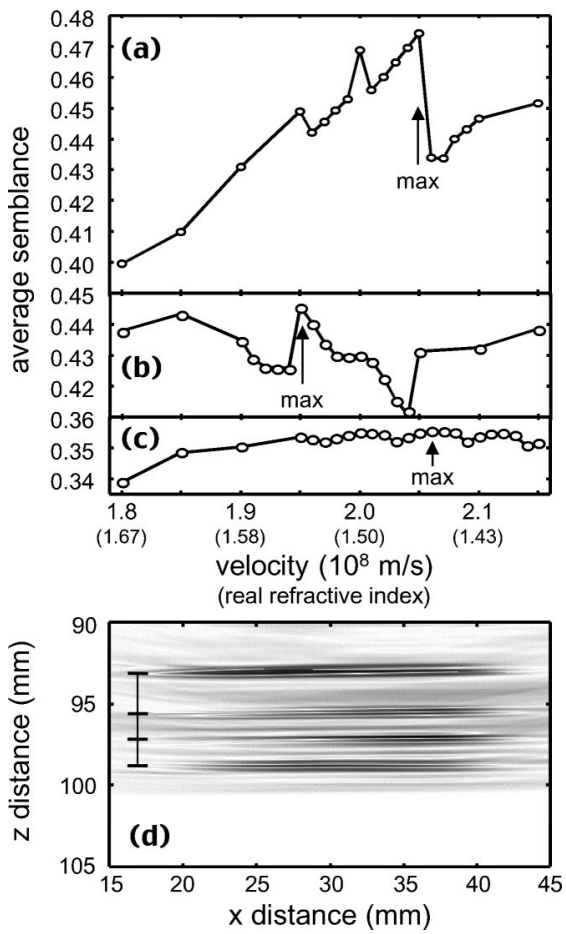

Fig. 10. The normalized semblance averages for the second, third, and fourth interfaces are shown in (a), (b), and (c), respectively. The semblance values were calculated for each $50-\mu \mathrm{m}$ wide column over an approximately 3 -mm-high window encompassing each interface. The average semblance for each velocity bin was taken over 30 columns. Unlike the results in Fig. 8, noise and multiple oscillations at the interfaces create a challenge for the semblance metric. The final Kirchhoff migration using the velocities taken from (a)-(c) is displayed in (d). The indicators, unlike those in Fig. 9(c), match the locations of the reconstructed interfaces.

for the semblance metric. Nevertheless, the velocities in each of the unknown regions are determined with surprising accuracy. The refractive indices of the upper and lower layers (both Teflon) are found to be 1.46, while the index of the middle layer (HDPE) is 1.54. These values compare favorably with the literature values of 1.43 and 1.52 for these two materials. ${ }^{29}$ From the widths of the semblance peaks in Figs. 10(a) and 10(b), we estimate an uncertainty in the determined velocities of roughly $\pm 0.5 \times 10^{7} \mathrm{~m} / \mathrm{s}$, corresponding to an uncertainty in refractive index of approximately \pm 0.04 for these materials. It is clear from the arrows in Figs. 10(a)-10(c) that this margin is sufficient to distinguish between the two very similar materials used in this composite target. Using the determined values for the velocities in each region, we can compute a revised image by Kirchhoff migration. This image is displayed in Fig. 10(d). We note that, unlike the uncorrected image in Fig. 9, the layer spacing in Fig. 10(d) is quite accurate. The positions of each of the buried interfaces (i.e., the thicknesses of the layers) are determined to within $\sim 10 \%$. The accuracy is limited by the coherence length of the $\mathrm{THz}$ pulse, as described above.

\section{CONCLUSIONS}

We have described a new image acquisition procedure using a fiber-coupled $\mathrm{THz}$ time-domain spectroscopy system.
Multistatic $\mathrm{THz}$ time-domain spectroscopy data acquisition allows more complex targets to be analyzed than those permitted by traditional approaches. This arrangement emulates the techniques of seismic imaging and borrows data processing methods from this mature field. The resulting images provide detailed information about the targets with the use of a simple and easily implemented algorithmic approach. The image reconstruction techniques provide quantitative information about both the thickness and the refractive index of the components of a composite target. It can be used to analyze buried interfaces through an iterative approach in which the velocity in any particular layer is determined by using the velocity of the preceding layer(s). Because of the large data redundancy, as well as the fact that migration relies primarily on pulse travel times rather than amplitude information, this imaging method is more robust against noise than many other multistatic imaging techniques.

To demonstrate these procedures, we simulated seismic data collection configurations on an optical bench by using $\mathrm{THz}$ time-domain spectroscopy, and we used migration signal processing techniques for image formation. These basic techniques are well-known in the geophysics community and have many analogies to imaging methods developed for electromagnetic waves. The development of new imaging algorithms for geophysical prospecting is an active area of research. Although we do not attempt to expand the current geophysical imaging techniques, it is clear that the use of real-world data creates situations not generally modeled in computer simulations. These results demonstrate the viability of the $\mathrm{THz}$ system as a test bed for the exploration of new seismic processing methods involving more complex model systems. These first steps open the door for the $\mathrm{THz}$ system to probe more challenging media and morphologies and for the investigation of new or improved reconstruction algorithms. Examples of challenges for which the $\mathrm{THz}$ system could be useful include strongly scattering media or stratified samples containing dispersive layers. Both of these situations are encountered in geophysical prospecting, and both pose significant difficulties in image reconstruction.

\section{ACKNOWLEDGMENTS}

We acknowledge partial support for this work by the Environmental Protection Agency and the National Science Foundation. The assistance of J. Van Rudd and Matt Warmuth at Picometrix, Inc., is gratefully acknowledged.

The corresponding author, Daniel M. Mittleman, may be contacted by e-mail, daniel@rice.edu.

\section{REFERENCES}

1. B. B. $\mathrm{Hu}$ and M. Nuss, "Imaging with terahertz waves," Opt. Lett. 20, 1716-1718 (1995).

2. D. Mittleman, R. Jacobsen, and M. Nuss, "T-ray imaging," IEEE J. Sel. Top. Quantum Electron. 2, 679-692 (1996).

3. D. M. Mittleman, M. Gupta, R. Neelamani, R. G. Baraniuk, J. V. Rudd, and M. Koch, "Recent advances in terahertz imaging," Appl. Phys. B 68, 1085-1094 (1999). 
4. M. Brucherseifer, P. Haring Bolivar, H. Klingenberg, and H. Kurz, "Angle-dependent $\mathrm{THz}$ tomography characterization of thin ceramic oxide films for fuel cell applications," Appl. Phys. B 72, 361-366 (2001).

5. R. A. Cheville and D. Grischkowsky, "Time domain terahertz impulse ranging studies," Appl. Phys. Lett. 67, 19601963 (1995)

6. D. Mittleman, S. Hunsche, L. Boivin, and M. Nuss, "T-ray tomography," Opt. Lett. 22, 904-906 (1997).

7. M. Tonouchi, M. Yamashita, and M. Hangyo, "Terahertz radiation imaging of supercurrent distribution in vortexpenetrated $\mathrm{YBa}_{2} \mathrm{Cu}_{3} \mathrm{O}_{7-\delta}$ thin film strips," J. Appl. Phys. 87, 7366-7375 (2000).

8. S. Hunsche, M. Koch, I. Brener, and M. C. Nuss, "THz nearfield imaging," Opt. Commun. 150, 22-26 (1998).

9. Q. Chen, Z. Jiang, G. Xu, and X.-C. Zhang, "Near-field terahertz imaging with a dynamic aperture," Opt. Lett. 25, 1122-1124 (2000)

10. J. L. Johnson, T. D. Dorney, and D. M. Mittleman, "Enhanced depth resolution in terahertz imaging using phaseshift interferometry," Appl. Phys. Lett. 78, 835-837 (2001).

11. M. Herrmann, M. Tani, and K. Sakai, "Display modes in time-resolved terahertz imaging," Jpn. J. Appl. Phys. Part 1 39, 6254-6258 (2000).

12. J. V. Rudd, J. L. Johnson, and D. M. Mittleman, "Quadrupole radiation from terahertz dipole antennas," Opt. Lett. 25, 1556-1558 (2000).

13. J. V. Rudd, J. L. Johnson, and D. M. Mittleman, "Crosspolarized angular emission patterns from lens-coupled terahertz antennas,” J. Opt. Soc. Am. B 18, 1524-1533 (2001).

14. J. V. Rudd and D. M. Mittleman, "The influence of substrate lens design in terahertz time-domain spectroscopy," J. Opt. Soc. Am. B 19, 319-329 (2002).

15. A. Ruffin, J. Decker, L. Sanchez-Palencia, L. LeHors, J. Whitaker, T. Norris, and J. Rudd, "Time reversal and object reconstruction with single-cycle pulses," Opt. Lett. 26, 681683 (2001).

16. M. Born and E. Wolf, Principles of Optics, 7th ed. (Cambridge U. Press, New York, 1999).

17. T. D. Dorney, J. L. Johnson, J. V. Rudd, R. G. Baraniuk, W. W. Symes, and D. M. Mittleman, "Terahertz reflection imaging using Kirchhoff migration,” Opt. Lett. 26, 1513-1515 (2001).

18. J. Scales, Theory of Seismic Imaging (Springer-Verlag, Berlin, 1995).
19. M. Dobrin and C. Savit, Introduction to Geophysical Prospecting, 4th ed. (McGraw-Hill, New York, 1988).

20. G. W. Purnell, "Observations of wave velocity and attenuation in two-phase media," Geophysics 51, 2193-2199 (1986).

21. J.-M. Ass'ad, T. M. Kusky, J. A. McDonald, and R. H. Tatham, "Implications of scale model seismology in the detection of natural fractures and microcracks," J. Seismic Explor. 1, 61-76 (1992).

22. C. Macdonald, P. M. Davis, and D. D. Jackson, "Inversion of reflection traveltimes and amplitudes," Geophysics 52, 606-617 (1987).

23. W. A. Schneider, "Integral formulation for migration in two and three dimensions," Geophysics 43, 49-76 (1978).

24. K. Waters, Reflection Seismology: A Tool for Energy Resource Exploration (Wiley, New York, 1981).

25. R. McGowan, R. Cheville, and D. Grischkowsky, "Experimental study of the surface waves on a dielectric cylinder via terahertz impulse radar ranging," IEEE Trans. Microwave Theory Tech. 48, 417-422 (2000).

26. R. Sullivan, Microwave Radar: Imaging and Advanced Concepts (Artech House, Norwood, Mass., 2000).

27. M. Brühl, J. Vermeer, and M. Kiehn, "Fresnel zones for broadband data," Geophysics 61, 600-604 (1996).

28. R. E. Sheriff, Geophysical Methods (Prentice-Hall, Englewood Cliffs, N.J., 1989)

29. J. R. Birch, J. D. Dromey, and J. Lesurf, "The optical constants of some common low-loss polymers between 4 and 40 $\mathrm{cm}^{-1}$," Infrared Phys. 21, 225-228 (1981)

30. C. H. Green, "Velocity determinations by means of reflection profiles," Geophysics 3, 295-305 (1938).

31. W. W. Symes and M. Kern, "Inversion of reflection seismograms by differential semblance analysis: algorithm structure and synthetic examples," Geophys. Prospect. 42, 565-614 (1994).

32. R. Versteeg, "The Marmousi experience: velocity model determination on a synthetic complex data set," Leading Edge 13, 927-936 (1994).

33. M. T. Taner and F. Koehler, "Velocity spectra: digital computer derivation and applications of velocity functions," Geophysics 34, 859-881 (1969).

34. N. S. Neidell and M. T. Taner, "Semblance and other coherency measures for multichannel data," Geophysics 36, 482497 (1971).

35. C. H. Dix, "Seismic velocities from surface measurements," Geophysics 20, 68-86 (1955). 